The Astrophysical Journal, 285:595-600, 1984 October 15

(C) 1984. The American Astronomical Society. All rights reserved. Printed in U.S.A.

\title{
IUE OBSERVATIONS OF THE CLUSTERS OF THE MAGELLANIC CLOUDS
}

\author{
J. G. COHEN ${ }^{1}$ AND R. M. RICH ${ }^{1}$ \\ Department of Astronomy, California Institute of Technology \\ AND \\ S. E. PERSSON ${ }^{1}$ \\ Mount Wilson and Las Campanas Observatories of the Carnegie Institution of Washington \\ Received 1984 January 25 ; accepted 1984 May 2
}

\begin{abstract}
$I U E$ observations of 17 clusters in the Large Magellanic Cloud are presented. The derived ultraviolet colors become progressively redder as the cluster's classification in the scheme of Searle, Wilkinson, and Bagnuolo increases. With the aid of theoretical models of the integrated light of coeval clusters of varying ages, we show that the SWB classification is one of increasing age accompanied by decreasing metallicity toward later SWB classes. The behavior of the UV absorption line features in the integrated light of the LMC clusters supports this contention.
\end{abstract}

Subject headings: clusters: globular — galaxies: Magellanic Clouds - ultraviolet: spectra

\section{INTRODUCTION}

The clusters of the Magellanic Clouds are close enough that color-magnitude diagrams and spectra of individual stars can be obtained, yet sufficiently distant that the brightness through reasonably sized apertures permits studies of their integrated light. The color-magnitude diagrams available to date, reviewed by Hodge (1983), indicate that the populous clusters of the Magellanic Clouds have a wide range in age, from those with blue and red supergiants less than $10^{8}$ years old to objects more than $10^{10}$ years old which resemble galactic globular clusters. Since it will be impossible to obtain such detailed observations of more distant stellar aggregates (such as galaxies or star clusters in them), it is important to find and calibrate parameters of the integrated light which can be used as age discriminants. Such indicators, which should also be insensitive to metallicity variations, must rely on visual or ultraviolet wavelengths, as the behavior of the composite light in the near-infrared is quite complicated (see Persson et al. 1983). Searle, Wilkinson, and Bagnuolo (1980, henceforth SWB) set up a ranking scheme based on two reddening-free indices derived from visual spectrophotometry of the integrated light of the clusters of the Magellanic Clouds. The locus of the observed points in the index-index plane defined a nonmonotonic but smooth curve with little dispersion. They separated this curve into seven segments, denoted SWB classes I to VII. Although they claimed only that the sequence is one of age and metallicity varying together in some (at that time) unknown way, it has been widely assumed that the SWB class division is one of age (see, for example, Rabin 1982 or Cohen 1982).

Ultraviolet colors are far more sensitive to age than visual colors, which more than compensates for their greater sensitivity to metallicity. We therefore observed a large sample of clusters in the LMC with the IUE satellite (§ II) and compare their UV colors with models of the integrated light ( $\$$ IV) to demonstrate that the SWB classification system is in fact an age-ranking scheme for younger clusters. We further show that with the possible exception of the oldest clusters, where the

\footnotetext{
${ }^{1}$ Guest Observer with the International Ultraviolet Explorer Observatory.
}

growing importance of the blue horizontal branch may cause anomalies, UV colors change monotonically with age. A brief discussion of the behavior of the absorption features in the spectra of the LMC clusters is presented in $\S \mathrm{V}$.

\section{OBSERVATIONS}

Seventeen clusters of the Large Magellanic Cloud and one of the Small Magellanic Cloud were observed with the IUE satellite in 1982 June and 1983 March. The large aperture, $10^{\prime \prime} \times 20^{\prime \prime}$ in size, was centered on brightest visual part of each cluster as seen with the IUE finder. The low-resolution short and long wavelength cameras were used. (See Boggess et al. $1978 a, b$ for a complete description of the instrument.) Five of the clusters were observed twice with each camera. Most of the spectra are well exposed, with at least 80 net data numbers/ pixel in the continuum, but for a few of the reddest clusters, even three hour exposures yielded spectra of only moderate signal-to-noise ratio. Examples of the spectra are shown in Figure 1.

The spectra were processed on the VAX 11/780 computer of the California Institute of Technology. Line by line spectra were used, with the background chosen to be free of cosmic-ray hits and microphonics. Hits were also cleaned from the resulting spectra, as was the reseau mark at $1780 \AA$ A. A 3 pixel boxcar smoothing reduced the pixel-to-pixel noise without degrading the actual spectral resolution. The individual observations were averaged for objects with multiple spectra if the spectra were of equal quality; otherwise the better spectrum was used.

Regions $50-100 \AA$ wide were chosen in which there were no extremely strong spectral features. The average flux per unit wavelength $F_{\lambda}$ was calculated within each interval and the results are listed in Table 1 . The fluxes in the selected intervals agree to within 0.1 dex for objects with multiple observations. The agreement is good given the small aperture and possible centering errors.

Visual magnitudes for a circular aperture with area equivalent to the IUE long slit were obtained from the multiple aperture photometry of Bernard and Bigay (1974). For the majority of clusters which did not have visual photometry at such small 


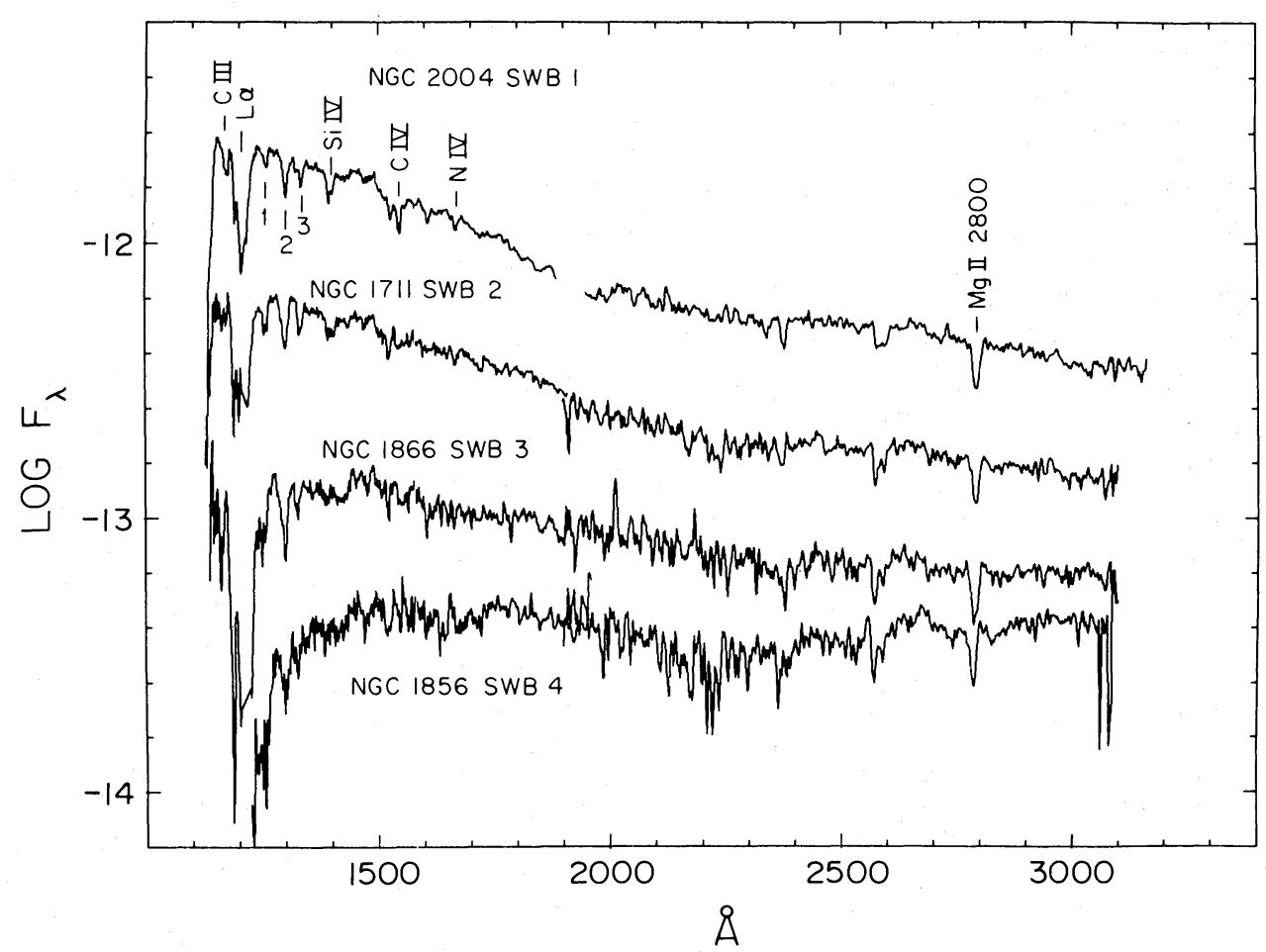

FIG. 1.-Representative IUE spectra of four LMC globular clusters. Identifications for some of the strongest features are marked.

apertures, visual magnitudes were calculated by extrapolating larger aperature published photometry (Bernard and Bigay 1974; Bernard 1975) using unpublished growth curves. These growth curves, kindly provided by Searle, were obtained with the $g$ filter of Thuan and Gunn (1976). The fraction of the total visible light in such a small aperture is low, ranging from $15 \%$ to $50 \%$. All ultraviolet-visual colors must be regarded as uncertain by roughly $30 \%$ due to possible differences in centering on these clumpy and extended clusters.
III. OBSERVATIONAL RESULTS

The clusters were selected to cover the full range in type in the system defined by SWB and to be as bright as possible. We accept as an initial premise the assertion by SWB that their classes primarily measure cluster age. Thus we plot in Figure 2 the difference in $\log \left(F_{\lambda}\right)$ between continuum regions centered at $1345 \AA$ and at $3150 \AA$ as a function of SWB class. For the four clusters not observed by SWB, the correlations of Persson

TABLE 1

OBSER VED FLUXES OF LMC CluSTERS

\begin{tabular}{|c|c|c|c|c|c|c|c|c|}
\hline \multirow{2}{*}{$\begin{array}{l}\text { ОВJECT } \\
\text { (NGC) }\end{array}$} & \multirow[b]{2}{*}{ SWB } & \multicolumn{7}{|c|}{$\left\langle-\right.$ LOG $\left.\left(F_{\lambda}\right)\right\rangle$ IN SELECTED INTERVALS ${ }^{a}$} \\
\hline & & $1330-1360$ & $1470-1520$ & $1730-1770$ & $1820-1900$ & $2640-2700$ & $2900-3000$ & $3100-3200$ \\
\hline 330(SMC) ...... & I & 12.12 & 12.17 & 12.31 & 12.38 & 12.66 & 12.75 & 12.79 \\
\hline $1711 \ldots \ldots \ldots \ldots$ & II & 12.27 & 12.30 & 12.44 & 12.50 & 12.74 & 12.82 & 12.85 \\
\hline $1755 \ldots \ldots \ldots \ldots$ & II-III & 12.40 & 12.41 & 12.53 & 12.60 & 12.77 & 12.84 & 12.88 \\
\hline $1774 \ldots \ldots \ldots \ldots$ & II & 12.39 & 12.42 & 12.54 & 12.61 & 12.89 & 12.99 & 13.05 \\
\hline $1805 \ldots \ldots \ldots \ldots$ & $\ldots$ & 12.20 & 12.27 & 12.41 & 12.48 & 12.76 & 12.88 & 12.90 \\
\hline $1818 \ldots \ldots \ldots \ldots$ & I & 12.04 & 12.12 & 12.25 & 12.32 & 12.61 & 12.69 & 12.73 \\
\hline $1850 \ldots \ldots \ldots \ldots$ & $\ldots$ & 12.41 & 12.41 & 12.51 & 12.57 & 12.76 & 12.82 & 12.86 \\
\hline $1854 \ldots \ldots \ldots \ldots$ & II & 12.94 & 12.93 & 13.02 & 13.08 & 13.26 & 13.35 & 13.32 \\
\hline $1856 \ldots \ldots \ldots \ldots$ & IV & 13.47 & 13.34 & 13.33 & 13.36 & 13.37 & 13.38 & 13.30 \\
\hline $1866 \ldots \ldots \ldots \ldots$ & III & 12.91 & 12.86 & 12.99 & 13.02 & 13.15 & 13.20 & 13.22 \\
\hline $1951 \ldots \ldots \ldots \ldots$ & $\ldots$ & 11.96 & 12.04 & 12.15 & 12.23 & 12.47 & 12.56 & 12.60 \\
\hline $1978 \ldots \ldots \ldots \ldots$ & VI & $\ldots$ & 14.93 & 14.40 & 14.62 & 14.29 & 14.28 & 14.01 \\
\hline $2004 \ldots \ldots \ldots \ldots$ & I & 11.79 & 11.89 & 12.02 & 12.12 & 12.35 & 12.46 & 12.52 \\
\hline $2019 \ldots \ldots \ldots \ldots$ & VII & 14.15 & 14.17 & 14.40 & 14.12 & 13.82 & 13.75 & \\
\hline $2041 \ldots \ldots \ldots \ldots$ & III & 12.65 & 12.70 & 12.80 & 12.85 & 13.06 & 13.12 & 13.17 \\
\hline $2100 \ldots \ldots \ldots \ldots$ & I & 12.38 & 12.37 & 12.41 & 12.48 & 12.56 & 12.62 & 12.66 \\
\hline $2157 \ldots \ldots \ldots \ldots$ & $\ldots$ & 12.71 & 12.69 & 12.79 & 12.86 & 13.03 & 13.06 & 13.11 \\
\hline $2210 \ldots \ldots \ldots \ldots$ & VII & 14.71 & 14.57 & 14.46 & 14.31 & 13.90 & 13.86 & 13.82 \\
\hline
\end{tabular}

${ }^{a}$ Ergs $\mathrm{cm}^{-2} \mathrm{~s}^{-1} \AA^{-1}$. 


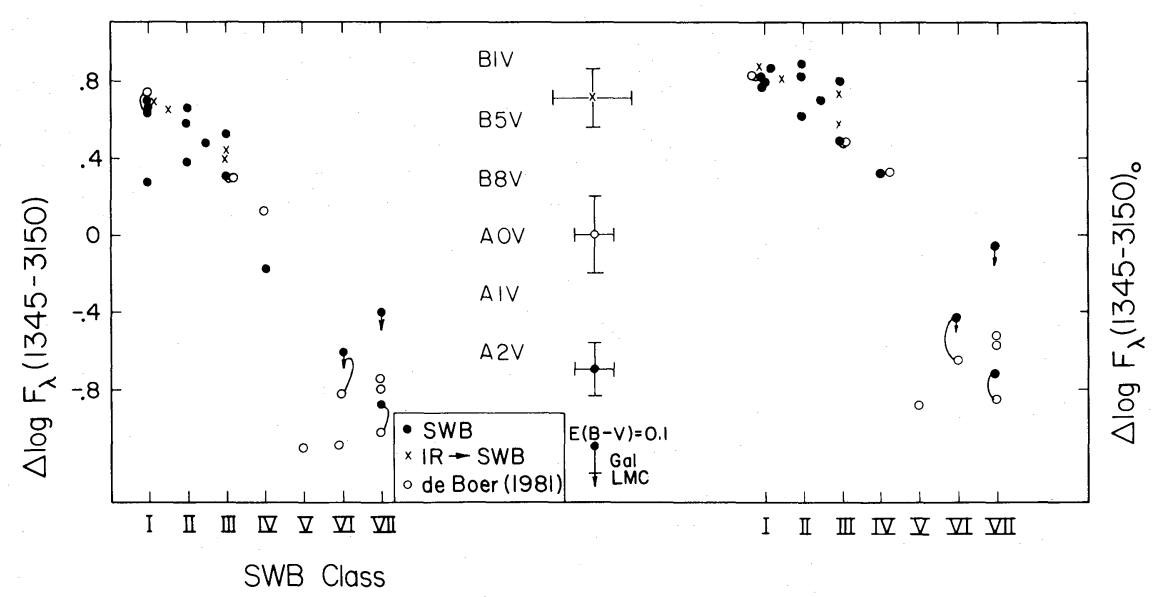

FIG. 2. - The average value of $\log \left(F_{\lambda}\right)$ in a $30 \AA$ interval centered at $1345 \AA$ minus the average value of $\log \left(F_{\lambda}\right)$ in a $100 \AA$ interval centered at $3150 \AA$ as a function of SWB class for clusters of the Magellanic Clouds. Filled circles denote those clusters classified by SWB, while the crosses denote clusters whose SWB classes have been inferred from visual and infrared photometry. Open circles represent IUE observations from de Boer (1981): the clusters in common are connected by solid lines to our data. Error bars for each type of point are shown in the middle of the figure. The left panel of the figure shows the observed UV colors; the right side displays the reddening-corrected UV colors. A reddening vector is drawn for $E(B-V)=0.1 \mathrm{mag}$. Its full length is appropriate for the LMC extinction law, while the length down to the horizontal bar is that for the galactic extinction law. Mean colors of solar metallicity low-reddening main-sequence stars are shown for various spectral types in the middle of the figure.

et al. (1983) between SWB class and infrared and visual colors of the integrated light of a large sample of Magellanic Cloud clusters were used to assign SWB classes. These are regarded as uncertain by one class in the SWB system and are denoted in Figure 2 by crosses. The open circles denote $I U E$ observations by de Boer (1981) plus one cluster from Cacciari and Fusi Pecci (1981). Data for clusters which were observed by both de Boer and by us are connected by a solid line. The error bars associated with the three different symbols and the colors of mainsequence stars with metallicities near that of the Sun and with low reddening $[E(B-V)$ less than $0.1 \mathrm{mag}]$ taken from the $I U E$ Spectral Atlas (Wu et al. 1981) are indicated in the centre of the figure. The excellent agreement of our UV colors with those of de Boer (1981) for the four clusters in common indicates that the error bars of Figure 2 may be overestimated.

In the right-hand part of Figure 2 we correct the data for extinction using individual values $E(B-V)$ for each cluster taken from Persson et al. (1983). It was assumed that the foreground galactic extinction corresponds to an $E(B-V)$ of 0.07 mag together with the appropriate galactic curve, while the remainder arises within the $\mathrm{LMC}$, for which the extinction law of Koornneef and Code (1981) was used. Because of the steeper far-UV extinction in the LMC, uncertainties of up to $0.2 \mathrm{mag}$ in dereddened colors $\left[0.08\right.$ in $\left.\log \left(F_{\lambda}\right)\right]$ are to be expected. A decrease in $E(B-V)$ by 0.1 mag produces the change in color shown by the full length of the arrow if the LMC extinction law is used and by the part of the arrow down to the horizontal bar if the galactic law is used. Note that because of the uncertainty in the extinction law for the LMC, it does not seem profitable at this time to attempt to derive reddening free parameters (analogs of the $Q$ s of SWB) from our data.

Figure 2 shows a strong correlation between ultraviolet color and SWB class. Earlier type SWB clusters have progressively bluer ultraviolet colors. This supports SWB's assertion that their classes are in fact primarily measuring cluster age, since for younger clusters, the main-sequence turnoff becomes hotter and more luminous, and it is this region we expect to dominate the ultraviolet light. The scatter in Figure 2 is small, considering the possible errors introduced by superposed bright stars from the field of the Magellanic Clouds and by centering problems, although in most cases the short and long wavelength IUE spectra were taken consecutively, without repositioning the slit.

Figure 3 presents the difference in $\log \left(F_{\lambda}\right)$ between $1345 \AA$ and $V$. The filled circles represent our data; the open circles those of de Boer and of Cacciari and Fusi Pecci. The smaller symbols represent clusters whose $V$ magnitudes were derived by extrapolating a measurement at a larger aperture without a suitable growth curve. The crosses again represent clusters observed by us whose SWB class has been estimated from the infrared colors. In the center of the figure the colors of mainsequence stars of approximately solar metallicity and of low reddening derived from the IUE Spectral Atlas are shown. Error bars for the three types of points are again indicated in the center of the figure. In the right-hand side of the figure the data have been corrected for reddening as described above. The arrow indicates the effect of a change in $E(B-V)$ by 0.1 mag for the galactic and the LMC extinction law in a manner similar to Figure 2.

The qualitative trend of Figure 2 is maintained in Figure 3, with the earlier SWB types having bluer ultraviolet-visual colors. The composite nature of the UV light can be appreciated by comparing the location of clusters whose reddening corrected colors match those of the earliest B stars in Figures 2 and 3 ; in each case the corresponding SWB class is somewhat earlier for Figure 3 than for Figure 2.

\section{A MODEL FOR THE ULTRAVIOLET LIGHT}

A model has been constructed for the integrated light of clusters all of whose stars are coeval. The Yale isochrones (Ciardullo and DeMarque 1977) were used in a manner similar to that described in Aaronson et al. (1978), where models were presented for the visual and infrared spectral region of old clusters. The horizontal branch was moved in effective temperature and luminosity as the cluster age changes according to the calculations of Sweigart and Gross (1976). The asymptotic giant branch was assumed to reach only to the luminosity of the helium flash irrespective of cluster age. The neglect of more luminous AGB stars in younger clusters is not important in the ultraviolet, although critical at longer wavelengths 


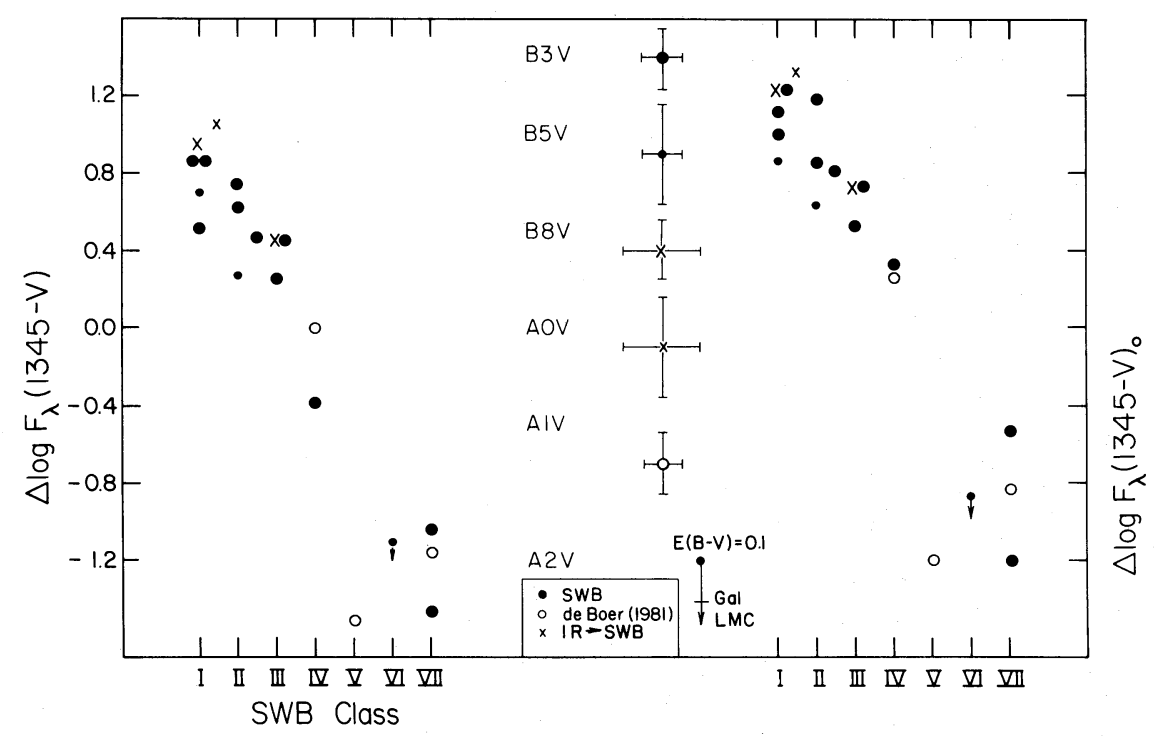

Fig. 3.-The same as Fig. 2 for the difference between the average flux in a $30 \AA$ interval centered at $1345 \AA$ and at $V$. The symbols are the same as in Fig. 2 . The large symbols represent those clusters with visual photometry at apertures bracketing the diameter equivalent to the IUE large slit, while the smaller ones denote clusters whose $V$ magnitude within the $I U E$ aperture has been extrapolated from photometry at larger apertures.

(Persson et al. 1983), as these stars are very red and do not contribute strongly to the integrated UV light. Stars in stages of evolution beyond the AGB are not included in these models.

The data in the IUE Spectral Atlas (Wu et al. 1981), supplemented by data for the later type stars from $A N S$ (Koornneef $e t$ al. 1982), for stars with low reddening were used to generate ultraviolet colors as a function of stellar effective temperature. These observed colors were used with the half solar isochrone to generate the integrated colors shown in Figure 4. The observed colors were corrected for the reduced line blanketing at lower metallicities using the difference in flux ratios from model stellar atmospheres of varying metallicities computed by Kurucz (1979). No abundance correction was made in colors of stars hotter than $10,000 \mathrm{~K}$, while a 1.5 dex change in $\log \left(F_{\lambda}\right)$ between $1345 \AA$ and $V$ occurs for the coolest stars for a metallicity decrease of a factor of 100 from solar. These substantial correction factors cannot be empirically verified, as the necessary set of observations of metal-poor stars in the UV does not exist. A single function of $T_{\text {eff }}$ was used, irrespective of luminosity class, to derive each color.

Figure 4 presents the results of these calculations for a Salpeter initial mass function throughout the entire mass range. The calibration of age versus SWB class is taken from Cohen (1982). The youngest age included in the Yale models is 200 million years, so that colors can be synthesized for SWB classes II-III and later only. Figure 4 is divided into two parts, corresponding to the $1350-3150$ A colors of the LMC clusters shown in Figure 2 and to the $1350-V$ colors of Figure 3. Colors are presented for three metallicities: half solar at ages from 200 billion years to 6 billion years, $1 / 20$ solar at ages from 1 billion to 6 billion years, and 1/200 solar at ages from 6 to 12 billion years. The reddening-corrected data for the LMC clusters from Figures 2 and 3 are superposed on the calculated colors.

The lowest metallicity calculations fit the SWB VII clusters well, but at these ages the exact treatment of the horizontal branch becomes quite important, as can be seen by the two branches of the 1/200 solar track in Figure 4. The first set of calculations includes a normal hot horizontal branch typical of the most metal-poor globular clusters in our Galaxy, while in the second set the horizontal branch was omitted entirely. Thus the exact number of horizontal branch stars and their temperature distribution critically affects the predicted ultraviolet colors for the older clusters. The half solar colors match well those of the clusters in SWB groups II to III, and the youngest clusters have colors consistent with their ultraviolet light being dominated by early B turnoff stars as well as blue supergiants. The slope of the relationship between UV color and SWB class I to IV is not matched well by an age variation alone which would correspond to the constant metallicity lines on Figure 4 that slope sharply downward. Nor are data matched by clusters of a fixed age, with metallicity decreasing as SWB class increases. Instead, a definite age-metallicity sequence is suggested as the proper analog of the SWB classes, with the later SWB classes being both older and more metal poor than the earlier ones. The predicted colors corresponding to the age-metallicity relationship derived by Cohen (1982) are shown as the thick solid line in each panel of Figure 4 . These provide an acceptable fit to the observed colors of the LMC clusters over the range covered by the models (ages between $2 \times 10^{8}$ and $12 \times 10^{9}$ years).

The most secure conclusion is that the SWB classes represent a sequence of metallicity decreasing as age increases, and the age calibration used here (which is that of Cohen 1982) is not grossly incorrect. The very young ages and large age ranges for SWB classes III to VI advocated by Hodge (1983) are ruled out by comparing the observed UV colors with even the crude theoretical integrated colors presented here. Further refinement of the age-metallicity calibration of the SWB classes requires a systematic study of the importance of post-AGB evolutionary phases and of UV colors in metal-poor stars.

The changes in UV colors as clusters age are dominated by the change in the relative flux emitted at the shortest wavelength $(1350 \AA$ here). Except perhaps for the very youngest clusters, even the hottest stars present have their peak emission longward of $1350 \AA$, thus the relative flux at $1350 \AA$ is a strongly increasing function of $T_{\text {eff }}$ at the main-sequence turnoff. It also appears to be true that the line blanketing is most severe at the shortest wavelengths. Thus to first order a 


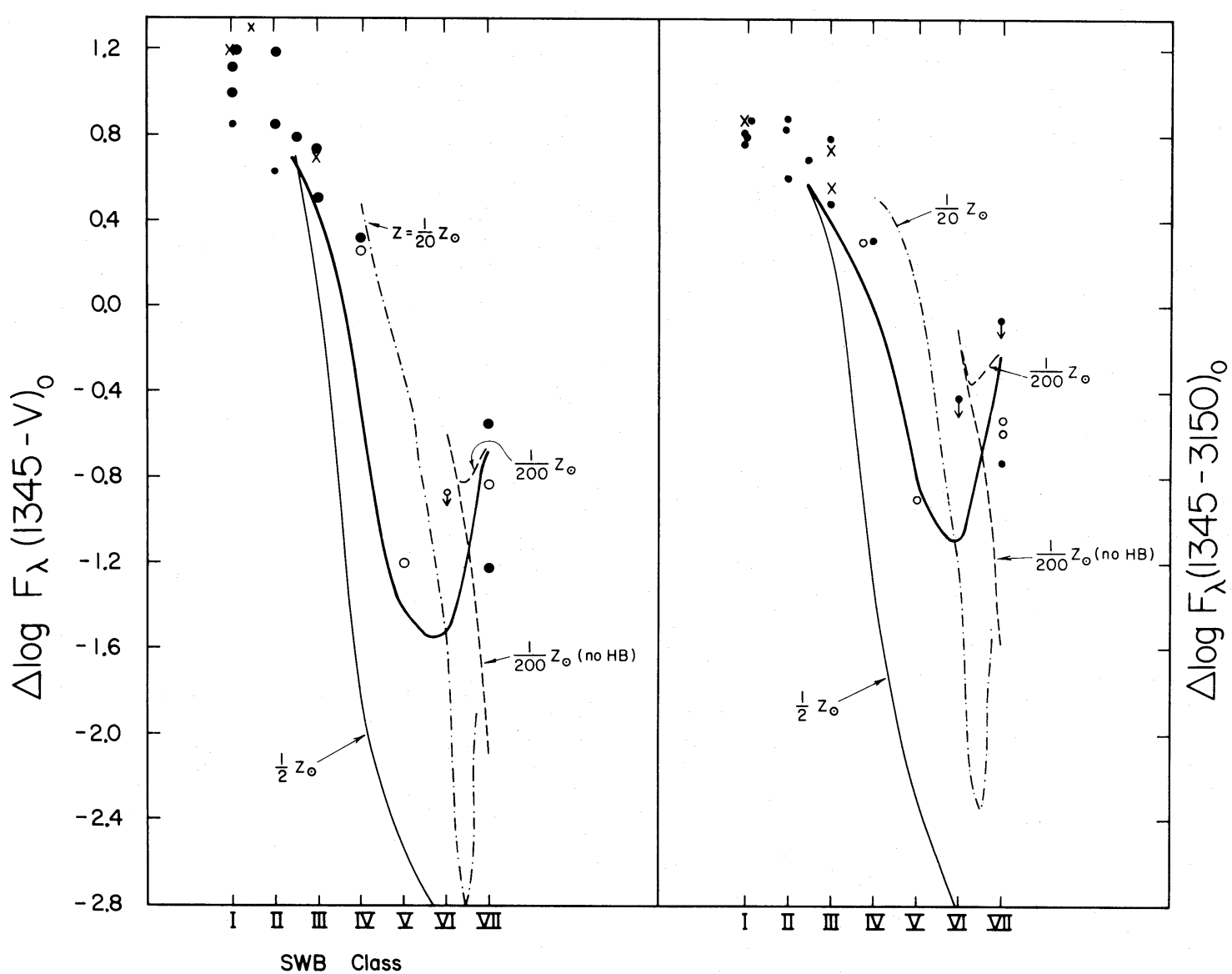

Fig. 4.-Predicted colors for $\Delta \log F_{\lambda}(1345-V)$ (left panel) and for $\Delta \log F_{\lambda}(1345-3150)$ (right panel) as a function of age (transformed to SWB class). The thin solid line represents half solar metallicity, the dot-dash line has $Z=1 / 20 Z_{\odot}$, and the dashed lines have $Z=1 / 200 Z_{\odot}$ with a blue horizontal branch, or with the $\mathrm{HB}$ omitted. Superposed on the theoretically predicted UV colors of the integrated light are the reddening-corrected UV colors of observed clusters in the Magellanic Clouds from Figs. 2 and 3. The symbols have the same meaning as in Fig. 3. The thick solid curves in each panel represent the colors predicted as a function of age using the age-metallicity relationship of Cohen (1982).

younger cluster has a flux distribution equivalent to that of a more metal-poor cluster. (A change in age of a factor of 5 mimics a reduction in metallicity from half solar to $1 / 200 Z_{\odot}$.) Given the uncertainties in the model colors, the small second order effects cannot be used to generate more complex combinations of colors between $1350 \AA$ and $V$ which could unequivocably separate age from metallicity variations.

\section{THE SPECTRAL FEATURES}

Many of the spectra of the MC clusters are of sufficiently high quality (as shown in Fig. 1) that the stronger absorption features are clearly detected. There is no sign of emission lines or P Cygni profiles in any of the cluster spectra. We have measured crude equivalent widths, where the choice of continuum is largely governed by the fact that the noise dominates over the weaker features. The continuum points were determined by smoothing the observed spectra over $50 \AA$ intervals. The uncertainty in the relative $W_{\lambda}$ between clusters is estimated to be $0.75 \AA$.

Figure 5 shows the three strongest features, the Si IV (1400 $\AA), C$ IV $(1550 \AA)$, and Mg II $(2800 \AA)$ doublets, as a function of SWB class for the LMC clusters with high precision IUE spectra. The two bluer features are shown only for the youngest clusters, as the SWP spectra for the later types do not have high enough signal-to-noise ratio to be used for this purpose.

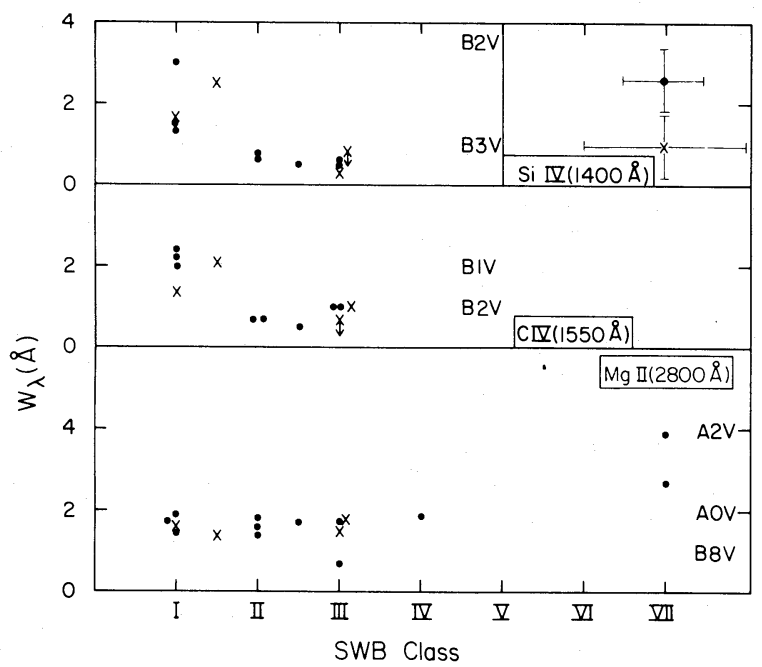

FIG. 5.-The behavior of the three strongest absorption features in the IUE spectra of Large Magellanic Cloud clusters as a function of SWB class. The symbols have the same meaning as in Fig. 2. Typical error bars for the two types of points are shown in the upper panel; the line strengths of solar metallicity main-sequence stars of various spectral types are shown at the right edge of each panel. 
The equivalent widths of main-sequence stars of solar metallicity as measured from the IUE Spectral Atlas (Wu et al. 1981) for Mg II and as given by Panek and Savage (1976) for the C IV and $\mathrm{Si}$ IV lines are indicated in each panel.

The expected trends of line strength with age can be seen in Figure 5. Those spectral features strongest in hot stars are strongest in the earliest SWB type clusters, while the $\mathrm{Mg}$ II doublet is strongest in the later SWB type clusters. To make this point more clearly, we rely on the compilation by Mermilliod (1981) of color-magnitude diagrams of the open clusters of the Galaxy. He has tabulated mean loci for clusters of various age groups from the Orion cluster to the Hyades. From the age calibration of the SWB classes of Cohen (1982), SWB classes I, II, and III should have main-sequence turnoffs at B1, B5, and A1, respectively. Since the light at $1400 \AA$ is dominated by the turnoff region in these clusters, the agreement between the line strength expected from the spectral type of the hottest stars present and the observed line strengths in the youngest LMC clusters is gratifying. However, Panek and Savage (1976) find that C IV (1550 $\AA)$ and Si IV (1400 $\AA$ ) become much stronger in supergiants. The contribution of the supergiants cannot be determined from our models for the integrated light.

A much wider range of stars contributes to the integrated light at $2800 \AA$. In the oldest clusters, the turnoff and giant branch dominate, while the contribution of the horizontal branch (for a blue HB in a metal-poor cluster) is only about $20 \%$ of the total. The observed line strengths of Mg II (2800 $\AA$ ) in the oldest LMC clusters are smaller than the $7 \AA$ that would be expected by roughly summing the contributions of the turnoff, giants, and HB. Although possible emission in the cores of the $\mathrm{Mg}$ II doublet has been ignored, the low observed $\mathrm{Mg}$ II line strengths are undoubtedly a reflection of the low metallicity of these old LMC clusters. The low metallicity deduced for the SWB class VII clusters is in agreement with the inferences of SWB and the spectroscopic results of Cohen (1982), Cowley and Hartwick (1982), and Rabin (1982). In this context it is interesting to note that the line strengths in NGC 330 (Si IV: $0.8 \AA, \mathrm{C}$ IV: $1.4 \AA, \mathrm{Mg}$ II: $\leq 1 \AA$ ), the only cluster observed in the SMC and hence omitted from Figure 5, are weaker than those of all the SWB class I clusters of the LMC.

\section{SUMMARY}

We have presented $I U E$ observations of 18 clusters in the Magellanic Clouds. In the UV the giants do not dominate the integrated light, unlike the situation in the visible and infrared, so that the impact of the giant branch shifting blueward as metallicity decreases is negligible. Furthermore, the shift in main-sequence location with metallicity is small compared to the large changes in its location as the cluster age varies. These effects are larger than the variation in ultraviolet color due to changes in line blanketing as a function of metallicity. Although our models for the integrated light of clusters of varying ages presented in $\S$ IV have several uncertainties and omissions, comparison of the theoretical colors predicted from the stellar evolutionary calculations of the Yale group with the $I U E$ data confirm that the SWB classification scheme represents an age-metallicity relationship with the youngest clusters having the earliest SWB types and being more metal rich. The age calibration of the SWB classes suggested by Cohen (1982) fits the data much better than that of Hodge (1983) for SWB classes III and later. Note that cluster UV colors are expected to be a monotonic function of age, until the very oldest clusters, where the horizontal branch increases in importance relative to the turnoff region. Variations in the HB then probably introduce some dispersion or change of slope into the UV color-age relationship.

The strongest absorption features in early-type stars are visible in the $I U E$ spectra. They vary with SWB class in a manner consistent with age being the dominant parameter of the SWB ranking scheme. The strength of the $\mathrm{Mg}$ II doublet at $2800 \AA$ in the LMC clusters of SWB class VII is weaker than would be expected from a crude synthesis of the composite light at that wavelength for old clusters. This confirms that the oldest clusters in the LMC are quite metal poor, a result already well established from spectra of individual stars.

We are grateful to NASA (grant NAG 5-63) for financial support and to the IUE Observatory staff for helpful advice.

\section{REFERENCES}

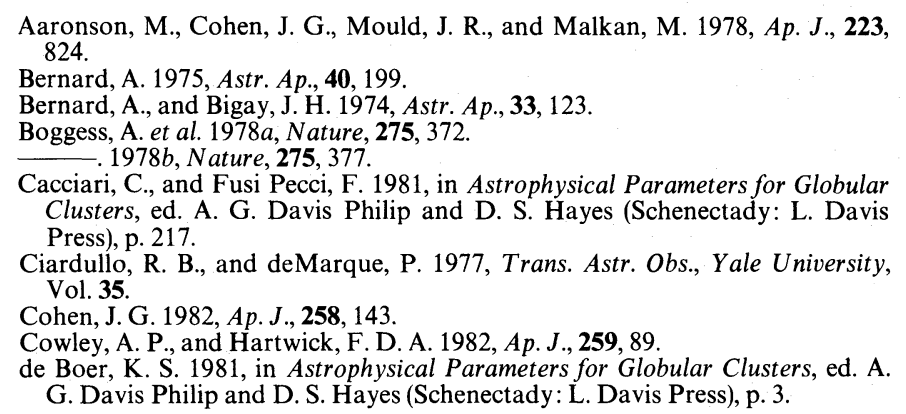

\author{
Hodge, P. W. 1983, Ap. J., 264, 470. \\ Koornneef, J., and Code, A. D. 1981, Ap. J., 247, 860 . \\ Koornneef, J., Meade, M. R., Wesselius, P. R., Code, A. R., and van Duinen, R. \\ J. 1982, Astr. Ap. Suppl., 47, 341. \\ Kurucz, R. L. 1979, Ap. J. Suppl., 40,1. \\ Mermilliod, J. C. 1981, Astr. Ap, 97, 235. \\ Panek, R. J., and Savage, B. D. 1976, Ap. J., 206, 167 \\ Persson, S. E., Aaronson, M., Cohen, J. G., Frogel, J. A., and Matthews, K. \\ 1983, Ap. J., 266, 105. \\ Rabin, D. 1981, Ap. J., 261, 85 \\ Searle, L., Wilkinson, A., and Bagnuolo, W. G. 1980, Ap. J., 239, 803 (SWB). \\ Sweigart, A. V., and Gross, P. G. 1976, Ap. J. Suppl., 32, 367 \\ Thuan, T. X., and Gunn, J. E. 1976, Pub. A.S.P., 88, 543. \\ Wu, C. C., Holm, A. V., Schiffer, F. H., III, and Turnrose, B. E. 1981, IUE \\ Spectral Atlas, N AS A IUE Newsletter, No. 14.
}

J. G. CoHEN and R. M. Rich: Department of Astronomy, California Institute of Technology, 105-24, Pasadena, CA 91125

S. E. Persson: Mount Wilson and Las Campanas Observatories, 813 Santa Barbara Street, Pasadena, CA 91101 